\title{
Structure of Individual versus Mixed Cat-Anionic Surfactants with Nanoparticles
}

\author{
Debes Ray ${ }^{1}$, Debasish Saha ${ }^{1}$, Vinod K. Aswal, a) and Joachim Kohlbrecher ${ }^{2}$ \\ ${ }^{1}$ Solid State Physics Division, Bhabha Atomic Research Centre, Mumbai 400 085, India \\ ${ }^{2}$ Laboratory for Neutron Scattering and Imaging, Paul Scherrer Institut, CH-5232 PSI Villigen, Switzerland \\ ${ }^{a)}$ Corresponding author: vkaswal@barc.gov.in
}

\begin{abstract}
Small Angle Neutron Scattering (SANS) experiments have been carried out to investigate the modification in the structure of nanoparticle and mixed cat-anionic surfactants complex with respect to individual surfactant (cationic or anionic). In absence of nanoparticles, individual surfactants (cationic DTAB or anionic SDS) self-assemble to form micelles and their mixed system forms large unilamellar vesicles. There is no physical interaction of anionic surfactant micelles with anionic silica nanoparticles, whereas cationic surfactant micelles are strongly adsorbed on anionic nanoparticles leading to aggregation. The vesicles of mixed surfactant system rearrange themselves to form a bilayer around the nanoparticles. Contrast variation SANS is employed to get the structural parameters of these multicomponents systems.
\end{abstract}

\section{INTRODUCTION}

Nanoparticles owing to their large surface to volume ratio and surface dependent properties result in a wide range of applications. Many of these applications require understanding of the interaction of nanoparticles with macromolecules such as surfactants. For example, interaction of surfactants with nanoparticles is utilized extensively for industrial and technical applications associated with colloidal stability, detergency and design of nanostructured functional interfaces [1,2]. The interaction of two components strongly depends on the characteristics of both the nanoparticle and surfactant used [3-6].

Surfactants are known to self-assemble to form nanosized micelles in aqueous solution [7]. In presence of nanoparticles, they can still form micelles or adsorb on nanoparticles or both. The resultant structure is expected to depend on how the micelle and nanoparticle interact. In the case similarly charged micelles, both nanoparticle and micelle coexist as individual. On the other hand for oppositely charged micelles, the strong adsorption of micelles on nanoparticle takes place. In this work, the interaction of mixed cat-anionic surfactants is examined with anionic nanoparticles to know if there is selectivity (strong adsorption of oppositely charged micelles) or new structures are formed. Contrast variation SANS experiments are carried out to determine the structure in these complexes under three different contrast conditions (i) both nanoparticles and micelles are visible, (ii) only micelles are visible and (iii) only nanoparticles are visible.

\section{EXPERIMENTAL}

Electrostatically stabilized $30 \mathrm{wt} \%$ colloidal suspension of silica nanoparticles (Ludox LS30) and the surfactants (DTAB and SDS) were obtained from Sigma Aldrich. Samples were prepared by dissolving weighted amount of silica and surfactants in $\mathrm{D}_{2} \mathrm{O} / \mathrm{H}_{2} \mathrm{O}$. The pure components (nanoparticles and surfactant) are examined for samples prepared in $\mathrm{D}_{2} \mathrm{O}$ because of high contrast and low incoherent background. For nanoparticle-surfactant complexes, nanoparticles are contrast matched for $38 \%$ of $\mathrm{H}_{2} \mathrm{O}$ whereas micelles are contrast matched for $92.5 \%$ of $\mathrm{H}_{2} \mathrm{O}$ in $\mathrm{H}_{2} \mathrm{O} / \mathrm{D}_{2} \mathrm{O}$ solvent. SANS data were collected in the wave vector transfer $(\mathrm{Q}=4 \pi \sin (\theta / 2) / \lambda$, where $\theta$ is scattering 
angle and $\lambda$ is the neutron wavelength) range of 0.05 to $3 \mathrm{~nm}^{-1}$. Measurements were carried out for fixed concentration $(1 \mathrm{wt} \%)$ of silica nanoparticles and surfactants. Samples were held in HELMMA quartz cells having thicknesses 1 or $2 \mathrm{~mm}$. The temperature of the samples was kept constant at $30{ }^{\circ} \mathrm{C}$ for all the measurements. Data were corrected for background and empty cell contributions and normalized to absolute cross-sectional unit using standard procedure.

\section{DATA ANALYSIS}

SANS measures the coherent differential scattering cross-section per unit volume $(d \Sigma / d \Omega)$ as a function of $Q$, which can be written as [8]

$$
\frac{d \Sigma}{d \Omega}(Q)=n P(Q) S(Q)+B
$$

where $n$ is the number density. $P(Q)$ is intraparticle structure factor and $S(Q)$ is interparticle structure factor. $B$ is a constant term representing incoherent background.

$P(Q)$ depends on shape and size of the particle and for a sphere particle is given by

$$
P(Q)=\left[\frac{4}{3} \pi R^{3}\left(\rho_{p}-\rho_{s}\right) \frac{3 J_{1}(Q R)}{Q R}\right]^{2}
$$

where $R$ is particle radius. $\rho_{p}$ and $\rho_{\mathrm{s}}$ are scattering length densities of particles and solvent, respectively

$P(Q)$ for a spherical shell having radius $\mathrm{R}$ and thickness $\mathrm{t}$ can be written as

$$
P(Q)=n\left(\rho_{\text {shell }}-\rho_{s}\right)^{2}\left[\frac{4}{3} \pi R^{3} \frac{3 J_{1}(Q R)}{Q R}-\frac{4}{3} \pi(R+t)^{3} \frac{3 J_{1}[Q(R+t)]}{Q(R+t)}\right]^{2} \quad .
$$

$S(Q)$ for charged components has been calculated using mean spherical approximation [9]. In the case of mass fractal of aggregate system, it is given by

$$
S_{f}(Q)=1+\frac{1}{(Q R)^{D}} \frac{D \Gamma(D-1)}{\left[1+\frac{1}{(Q \xi)^{2}}\right]^{(D-1) / 2}} \sin \left\{(D-1) \times \tan ^{-1}(Q \xi)\right\}
$$

where $\xi$ signifies the maximum length up to which fractal microstructure exists, $R$ is the size of building block and $D$ is the fractal dimension.

In the analysis, the theoretical models are compared with the experimental data and parameters were optimized by means of nonlinear least-square fitting program.

\section{RESULTS AND DISCUSSION}

Figure 1(a) shows SANS data from 1wt\% pure surfactants (SDS and DTAB) and their mixed system (equal weight fraction) in $\mathrm{D}_{2} \mathrm{O}$. The data from pure components are typical that of concentrated charged particles having a correlation peak arising from $S(Q)$. The fitted structural parameters are given in Table 1. Both the surfactants form slightly elongated prolate ellipsoidal micelles. The charge on the SDS and DTAB micelles is found to be -17.8 and +9.5 e.u., respectively. The scattering is drastically enhanced in the low Q region when two surfactants are mixed. This is an indication of formation large structures. The data are fitted with the bilayer vesicles having radius $29.8 \mathrm{~nm}$ and thickness $2.8 \mathrm{~nm}$. The charge neutralization by the two oppositely charged surfactants gives rise to high packing parameter leading to micelles to vesicle transition.

SANS data of interaction of nanoparticles with individual components and mixed surfactants are given in Figure 1(b). In the case of SDS with nanoparticles, there is no much change in the scattering in the lower Q region but build up of additional scattering in the higher Q region (surfactant micelles) as compared to pure nanoparticles. The overall scattering is almost equal to the addition of two components suggesting no physical interaction of SDS micelles with nanoparticles (anionic-anionic repulsion). For DTAB and mixed (SDS+DTAB) surfactants, SANS data suggest the strong interaction with nanoparticles. The linear Q dependence in the low Q region for DTAB indicates the formation of fractal aggregates (micelle mediated nanoparticle aggregation) in the oppositely charged components system. The nice oscillations observed for the mixed (SDS+DTAB) surfactants arise because of the core-shell structure formed from some kind of decoration of surfactants on the nanoparticle [3-5]. 

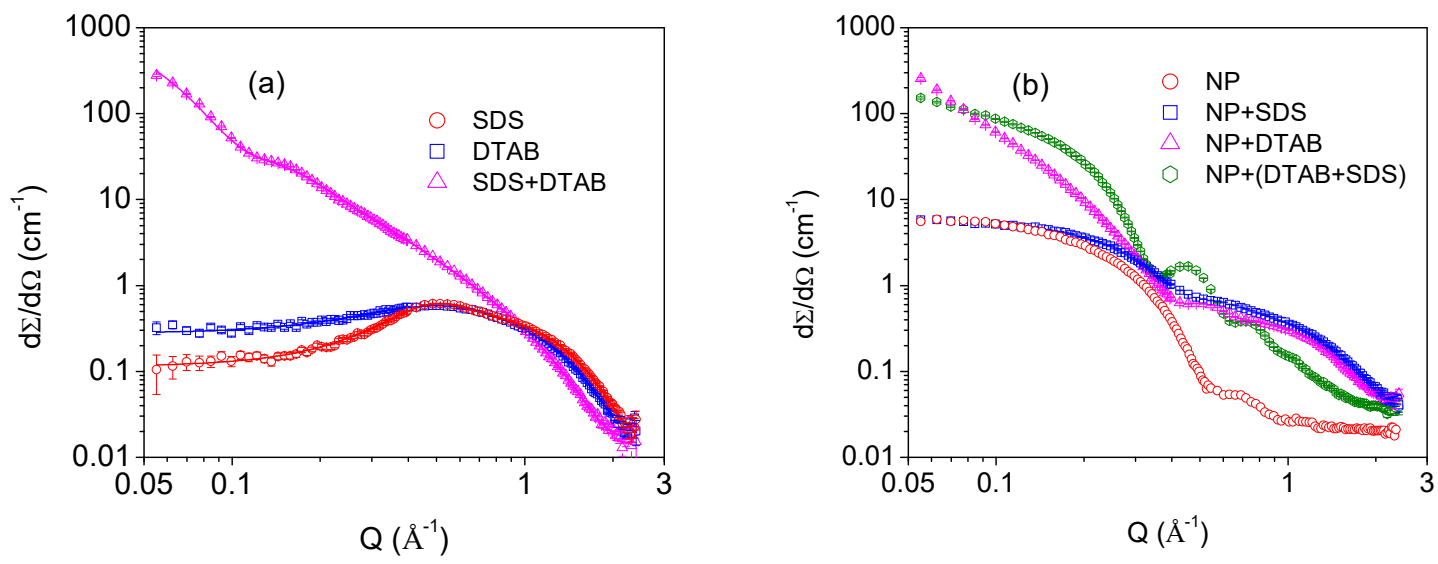

FIGURE 1. SANS Data from (a) $1 \mathrm{wt} \%$ pure surfactants (SDS and DTAB) and their mixed system, (b) nanoparticles and their interaction with surfactants (SDS and DTAB) and mixed system.

TABLE 1. Structural parameters of $1 \mathrm{wt} \%$ pure surfactants (SDS and DTAB) and their mixed system.

\begin{tabular}{lllll}
\hline System & Structure Morphology & Dimensions* & & Charge \\
\hline $1 \mathrm{wt} \%$ SDS & Ellipsoidal Micelle & $a=2.14 \mathrm{~nm}$ & $b=c=1.67 \mathrm{~nm}$ & -17.9 e.u. \\
$1 \mathrm{wt} \%$ CTAB & Ellipsoidal Micelles & $a=2.57 \mathrm{~nm}$ & $b=c=1.74 \mathrm{~nm}$ & $+9.5 \mathrm{e} . \mathrm{u}$. \\
$0.5 \mathrm{wt} \%$ SDS + & Bilayer Vesicles & $R=29.8 \mathrm{~nm}$ & $t=2.80 \mathrm{~nm}$ & - \\
$0.5 \mathrm{wt} \%$ CTAB & & & & \\
\hline
\end{tabular}

* $a=$ semimajor axis, $b=c=$ semiminor axis, $R=$ radius and $t=$ thickness

To simplify the analysis, the individual components in multi-components system are uniquely determined in the SANS by contrast matching the components. Figure 2(a) shows the data of nanoparticle-surfactant complexes when the surfactant is contrast matched. In this case, the nanoparticles are only visible. Data clearly confirm that the nanoparticles remain individual for SDS and (SDS+DTAB) mixed surfactants, whereas DTAB leads to the fractal aggregation of nanoparticles. The fractal aggregates are found to be mass fractal having fractal dimension of 2.5 [10].
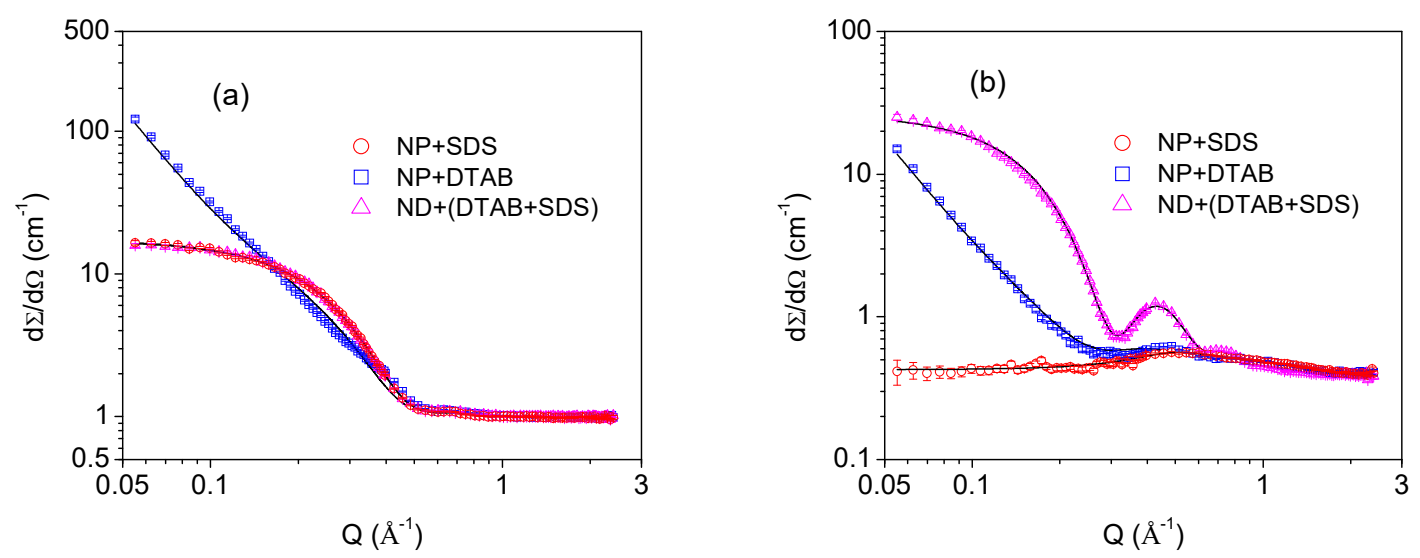

FIGURE 2. SANS Data from nanoparticles with surfactants (SDS and DTAB) and their mixed system under (a) surfactant contrast-matched and (b) nanoparticles contrast-matched conditions. 
Modification in the configuration of surfactant in the nanoparticle-surfactant complex is directly observed when the nanoparticles are contrast matched [Figure 2(b)]. In the case of SDS surfactant, the scattering is quite low and fit to that of individual micelles. For DTAB surfactant, the data are fitted with fractal aggregates of micelles adsorbed on nanoparticles. The data are fitted with core-shell structure having radius equal to that of nanoparticles $(8.2 \mathrm{~nm})$ and thickness equal to bilayer thickness $(2.8 \mathrm{~nm})$ that is formed in the mixed (SDS+DTAB) surfactant system. These results thus suggest that the interaction of ionic surfactant with charged nanoparticles is modified when the mixed surfactant are used. The attractive electrostatic interaction leads to the adsorption of surfactant on nanoparticles whereas mixed micellization (driven by hydrophobic interaction) determines the structure of surfactant adsorption.

\section{CONCLUSION}

SANS has been performed to examine the interaction of nanoparticles with individual cat-anionic surfactants (DTAB and SDS) and their mixed (DTAB+SDS) surfactants. In particular, contrast variation SANS is unique technique to study such multi-components systems to simplify the analysis. The combination of electrostatic interaction between nanoparticle and surfactant with hydrophobic interaction between oppositely charged surfactant controls the structure of surfactant adsorption of nanoparticles. It is shown that non-adsorbing surfactant when mixed with strongly adsorbed surfactant form a bilayer adsorption on nanoparticles.

\section{REFERENCES}

1. H. Hein, C. Pramanik, O. Heinz, Y. Ding, R.K. Mishra, D. Marchon, R.J. Flatt, I. Estrela-Lopis, J. Llop, S. Moya and R.F. Ziolo, Surf. Sci. Rep. 72, 1-58 (2017).

2. B. Kowalczyk, K.J.M. Bishop, I. Lagzi, D. Wang, Y. Wei, S. Han and B.A. Grzybowski, Nat. Mater. 11, 227-232 (2012)

3. D. Lugo, J. Oberdisse, M. Karg, R. Schweins and G. Findenegg, Soft Matter 5, 2928-2936 (2009).

4. K.P. Sharma, V.K. Aswal and G. Kumaraswamy, J. Phys. Chem. B 114, 10986-10994 (2010)

5. S. Kumar, V.K. Aswal and J. Kohlbrecher, Langmuir 28, 9288-9297 (2012).

6. D. Ray, S. Kumar, V. K. Aswal and J. Kohlbrecher, Langmuir 34, 259-267 (2018).

7. V.K. Aswal and P.S. Goyal, Phys. Rev. E 61, 2947-2953 (2000).

8. J.S. Pedersen, Adv. Colloid Interface Sci. 70, 171-210 (1997).

9. J.B. Hayter and J.Penfold, Colloid Polym. Sci. 261, 1022-1030 (1983)

10. S. Kumar, I. Yadav, V.K. Aswal and J. Kohlbrecher, Langmuir 34, 5679-5695 (2018) 